\title{
Comportamento Alimentar Inadequado em Adolescentes de Juiz de Fora
}

\author{
Leonardo de Sousa Fortes ${ }^{1}$ \\ Faculdade de Educação Física e Desporto da Universidade Federal de Juiz de Fora, \\ Juiz de Fora, Brasil \\ Ana Carolina Soares Amaral \\ Departamento de Educação Física do Instituto Federal de Ciência e Tecnologia \\ do Sudeste de Minas Gerais, Barbacena, Brasil \\ Maria Elisa Caputo Ferreira \\ Departamento de Fundamentos da Educação Física da Universidade Federal \\ de Juiz de Fora, Juiz de Fora, Brasil
}

\section{Resumo}

O objetivo do presente estudo foi avaliar os comportamentos alimentares de adolescentes do sexo feminino de Juiz de Fora/MG, comparando-os em função do sexo e do estado nutricional. Participaram 249 sujeitos com idade entre 10 e 15 anos. Utilizou-se o Eating Attitudes Test (EAT-26) para avaliar o comportamento alimentar. Aferiram-se peso e estatura para se calcular o índice de massa corporal (IMC). O estado nutricional foi obtido pelos pontos de corte do IMC. Conduziu-se análise multivariada de covariância para comparar os três fatores do EAT-26 em função do sexo e do estado nutricional. Os resultados demonstraram diferenças significativas nas subescalas Bulimia e preocupação com alimentos e Dieta de acordo com o estado nutricional $(\mathrm{p}<0,05)$. Concluiu-se que o comportamento alimentar foi diferente entre os grupos de estado nutricional.

Palavras-chave: Comportamento alimentar, adolescentes, transtornos alimentares.

\section{Inappropriate Eating Behavior of Adolescents in Juiz de Fora}

\begin{abstract}
The aim of this study was to evaluate the eating behaviors of female adolescents in Juiz de Fora/MG, comparing them selves according to state nutritional. Participants 249 adolescents aged between 10 and 15 years. We used the Eating Attitudes Test (EAT-26) to evaluate the inadequate eating behavior. We measured body weight and height to calculate the body mass index (BMI). Nutritional status was obtained by points BMI cutoff. Multivariate covariance analysis was conducted to compare the three factors of the EAT-26 according to nutritional status. The results showed significant differences in the subscales Bulimia and concern with food and Diet $(\mathrm{p}<.05)$ according to nutritional status. It was concluded that eating behavior was different between groups nutritional status.
\end{abstract}

Keywords: Eating behavior, adolescents, eating disorders.

Endereço para correspondência: Rua Hameleto Fellet, 20/201, Vale do Ipê, Juiz de Fora, MG, Brasil 36036130. E-mail: leodesousafortes@hotmail.com, acsamaral@hotmail.com e caputoferreira@terra.com.br Agência de Financiamento: Conselho Nacional de Desenvolvimento Científico e Tecnológico (CNPq). 


\section{El Comportamiento Inadecuado de Comer en Adolescentes de Juiz de Fora}

\section{Resumen}

El objetivo de este estudio fue evaluar las conductas alimentarias de los adolescentes en Juiz de Fora/ MG, comparándolos con base en el estado nutricional. Participantes 249 alumnos de edades comprendidas entre 10 y 15 años de sexo femenino. Se utilizó el Eating Attitudes Test (EAT-26) para evaluar la conducta alimentaria. Él midió el peso y la altura para calcular el índice de masa corporal (IMC). El estado nutricional se obtuvo mediante puntos de corte de IMC. Se realizó un análisis multivariado de covarianza para comparar los tres factores del EAT-26 en función del sexo y el estado nutricional. Los resultados mostraron diferencias significativas en las subescalas bulimia y preocupación por la comida y la dieta de acuerdo con el estado nutricional $(\mathrm{p}<.05)$. Se concluyó que el comportamiento de alimentación distinta entre grupos de estado nutricional.

Palabras clave: El comportamiento de alimentación, adolescentes, trastornos de la alimentación.

Segundo a Organização Mundial de Saúde (World Health Organization [WHO], 2007), adolescência é um período compreendido entre os 10 e 19 anos de idade. Nesta fase, o jovem vivencia mudanças psicológicas e sociais, além de passar por alterações morfológicas decorrentes da puberdade (Martins, Pelegrini, Matheus, \& Petroski, 2010; Scherer, Martins, Pelegrini, Matheus, \& Petroski, 2010). Durante esta eta$\mathrm{pa}$, as meninas geralmente apresentam aumento do percentual de gordura, ao contrário do sexo masculino, que costuma demonstrar aumento da massa livre de gordura (Siervogel et al., 2003).

Estas alterações podem acentuar a preocupação que o adolescente possui com o seu corpo. Estudos têm apresentado elevada prevalência de insatisfação corporal em jovens escolares brasileiros (Castro et al., 2010; Duca, Garcia, Sousa, Oliveira, \& Nahas, 2010; Scherer et al., 2010). Sabe-se que a depreciação com o peso e com a aparência física pode predispor os jovens a desenvolverem hábitos deletérios a saúde na tentativa de modificarem suas morfologias corporais (Miranda, Conti, Bastos, \& Ferreira, 2011; Oliveira \& Hutz, 2010), gerando comportamentos alimentares inadequados e outras práticas prejudiciais à saúde (Alvarenga, Scagliusi, \& Philippi, 2011; Ximenez, Colares, Bertulino, Couto, \& Sougey, 2011). Alguns autores ressaltam que a autoindução de vômitos, o uso de medicamentos (diurético e laxantes) para emagrecimento e a restrição alimentar são sintomas comuns de síndromes psicológicas como a anorexia nervosa (AN) e a bulimia nervosa (BN; Alvarenga et al., 2011; Bernardi, Harb, Levandovski, \& Hidalgo, 2009; Rossi, Moreira, \& Rauen, 2008).

Estudos têm demonstrado que o sexo feminino é mais acometido por influências negativas advindas da cultura e como consequência, apresenta maior risco para os transtornos alimentares (Alvarenga et al., 2011; Castro et al., 2010; Miranda et al., 2011; Rossi et al., 2008; Scherer et al., 2010). Investigações recentes têm comprovado que jovens do sexo feminino apresentam maior chance de desenvolverem comportamentos alimentares inadequados quando comparadas aos adolescentes do sexo masculino (Johnson et al., 2004; Santos, Tassitano, Nascimento, Petribu, \& Cabral, 2011).

Atualmente, são evidenciados resultados que demonstram o aumento da prevalência de hábitos alimentares deletérios a saúde em jovens brasileiros (Castro et al., 2010; Dunker, Fernandes, \& Carreira, 2009; Gomes, Legnani, Legnani, Gregorio, \& Souza, 2010; Martins et al., 2010; Oliveira \& Hutz, 2010; Rossi et al., 2008; Scherer et al., 2010). Parece que os comportamentos alimentares inadequados têm se tornado hábito cotidiano na vida de adolescentes (Oliveira \& Hutz, 2010). No entanto, o fato do indivíduo apresentar alguns comportamentos alimentares inapropriados não remete neces- 
sariamente a síndromes psicológicas como os transtornos alimentares, mas torna este sujeito mais vulnerável para o desenvolvimento de tais doenças (Martins et al., 2010; Rossi et al., 2008). Sabe-se que o sexo feminino e o jovem com o estado nutricional inadequado (sobrepeso/obesidade) costumam ser mais acometidos por estes comportamentos (Alvarenga et al., 2011; Scherer et al., 2010). No entanto, até o momento, não se identificou estudo comparando os fatores de atitudes alimentares em função do estado nutricional. Portanto, esta pesquisa destaca-se como inovadora ao propor este tipo de análise com os atributos do comportamento alimentar no Brasil. Diante do exposto, o objetivo do presente estudo foi avaliar os comportamentos alimentares de adolescentes do sexo feminino de Juiz de Fora/ MG, comparando-os de acordo com o estado nutricional.

\section{Método}

\section{Participantes}

Trata-se de pesquisa transversal realizada em escolas privadas e públicas, com adolescentes do sexo feminino, com idade entre 10 e 15 anos, residentes na cidade de Juiz de Fora/ MG. Segundo informações do Censo Escolar do ano de 2010, este município apresentava cerca de 25.000 (vinte e cinco mil) jovens nesta faixa-etária, matriculadas em escolas privadas e públicas. Para o cálculo amostral, adotou-se prevalência de $20 \%$ para o comportamento alimentar inadequado (Martins et al., 2010; Scherer et al., 2010), $5 \%$ de erro amostral e $95 \%$ de confiança, chegando-se ao tamanho amostral de 244 adolescentes.

A seleção amostral ocorreu aleatoriamente, por meio de sorteio simples, em duas etapas. Realizou-se primeiro o sorteio das escolas em cada região e, posteriormente, o sorteio das adolescentes nessas unidades. As escolas foram selecionadas valendo-se da relação fornecida pelo setor de estatística da Secretaria de Educação do Município de Juiz de Fora/MG. A amostra final da pesquisa foi distribuída em 8 pontos diferentes de coleta (escolas) e constituída por adolescentes do sexo feminino na faixa etária de
10 a 15 anos de idade, presentes nas escolas nos dias da coleta e cujos pais autorizaram a participação na pesquisa. Foram incluídas no estudo somente as jovens que apresentassem o Termo de Consentimento Livre e Esclarecido (TCLE) assinado pelo responsável e que estivessem regularmente matriculadas no ensino fundamental/ médio na cidade de Juiz de Fora/MG no ano de 2011. Ademais, excluíram-se da investigação as adolescentes que não aceitaram ser submetidos às atividades da pesquisa. Desta forma, definiu-se como unidade experimental para a presente investigação a adolescente do sexo feminino residente na cidade de Juiz de Fora/MG.

Participaram do estudo 270 jovens, sendo que 21 destas foram excluídas por não responderem aos questionários em sua totalidade e/ou não participarem das aferições antropométricas, obtendo-se então, uma amostra de 249 participantes. A média de idade foi de $12,72(D P=1,59)$ anos. Para a inclusão no estudo, a adolescente necessitava estar regularmente matriculada nas instituições de ensino fundamental/médio na cidade de Juiz de Fora/MG, ter disponibilidade para responder questionários e realizar medidas antropométricas, além de apresentar o TCLE assinado pelo responsável.

O projeto foi aprovado pelo Comitê de Ética e Pesquisa em Seres Humanos da Universidade Federal de Juiz de Fora, recebendo protocolo 2282.022.2011 com parecer número 022/2011, de acordo com a resolução 196/96 do Conselho Nacional de Saúde.

\section{Anamnese}

Eating Attitudes Test (EAT-26). Para avaliar o comportamento alimentar, utilizou-se o Eating Attitudes Test em sua versão abreviada (EAT-26; Garner, Olmsted, Bohr, \& Garfinkel, 1982). Este é um instrumento de autopreenchimento com 26 questões em escala do tipo likert variando de 0 (nunca) a 3 (sempre) pontos, sendo que o item 25 apresenta escore invertido $(0=$ sempre, muitas vezes e as vezes; $1=$ poucas vezes; 2 = quase nunca; 3 =nunca). Estas questões buscam avaliar a dieta, os sintomas de bulimia, a restrição alimentar e a influência do ambiente sobre os hábitos alimentares, e estão distribuídas 
em três fatores: (a) dieta - diz respeito à recusa patológica a alimentos com alto teor calórico e preocupação com aparência física; (b) bulimia e preocupação com os alimentos - refere-se a episódios de compulsão alimentar, seguido por comportamentos purgativos para perda/controle de peso corporal e; (c) autocontrole oral - reflete o autocontrole em relação à comida e avalia forças ambientais e sociais estimulantes à ingestão alimentar. Escore maior ou igual a 20 indica riscos para os transtornos alimentares (Garner et al., 1982). O EAT-26 foi validado no Brasil para adolescentes do sexo feminino (Bighetti, Santos, Santos, \& Ribeiro, 2004), no qual apresentou alpha de Cronbach de 0,82. Para a presente amostra, calculou-se a consistência interna, identificando-se valor de 0,89 .

Avaliação Antropométrica. A massa corporal foi mensurada por uma balança digital portátil da marca Tanita com precisão de $100 \mathrm{~g}$ e capacidade máxima de $200 \mathrm{~kg}$. Utilizou-se estadiômetro portátil com precisão de $0,1 \mathrm{~cm}$ e altura máxima de 2,20m da marca Welmy para mensurar a estatura das adolescentes.

$\mathrm{O}$ índice de massa corporal $[\mathrm{IMC}=$ massa corporal $(\mathrm{kg}) /$ estatura $\left(\mathrm{m}^{2}\right)$ ] foi usado como indicador de estado nutricional. A classificação do IMC obedeceu aos critérios da Organização Mundial de Saúde (WHO, 2007), que propõe a classificação segundo os percentis em função da idade cronológica: baixo peso (percentil 5), peso normal (percentil 5 a 85), sobrepeso (percentil 85 a 95) e obeso (percentil 95).

\section{Procedimentos}

Inicialmente, a direção de cada escola foi contatada pelos pesquisadores que explicaram os objetivos e os procedimentos do projeto. Após aprovação inicial, distribuiu-se o TCLE as alunas que foram esclarecidas sobre todos os procedimentos necessários para a realização da pesquisa.

Os dados foram coletados entre os meses de março e junho de 2011, sendo realizadas duas etapas. No primeiro momento, em sala de aula, após a entrega do TCLE devidamente assinado pelos responsáveis, as alunas responderam individualmente o EAT-26 e a um cabeçalho de identificação contendo a idade. Os questionários foram aplicados por um mesmo pesquisador, que padronizou as orientações verbais. As participantes não se comunicaram entre si e não houve limite de tempo para o preenchimento do EAT-26.

Com a entrega do questionário, em um segundo momento, a jovem foi encaminhada para uma sala cedida pela escola para mensuração de peso e estatura. Para tanto, esta adolescente deveria estar trajando uniforme para a aula de Educação Física e descalça.

\section{Análise Estatística}

Utilizaram-se medidas de tendência central (média), dispersão (desvio padrão) e frequências para a descrição das variáveis do estudo. Conduziram-se análises univariadas de covariância (ANCOVA) para comparar os três fatores (dieta; bulimia e preocupação com os alimentos; autocontrole oral) e o escore total do EAT-26 de acordo com o estado nutricional (covariável idade). Além disso, a regressão logística binária foi utilizada para analisar a razão de chances para os comportamentos alimentares inadequados (EAT-26 $\geq 20$ ) em razão das classificações do estado nutricional. Todos os dados foram tratados no software SPSS versão 17.0, adotando-se nível de significância de $5 \%$.

\section{Resultados}

Participaram da pesquisa 249 meninas, sendo que 142 (57\%) estavam matriculadas em instituições públicas e 107 (43\%) eram pertencentes às escolas particulares. A média de idade de $12,72(D P=1,59)$. O IMC derivado do peso e estatura aferidos foi de 19,77 $(D P=3,66)$. Ademais, os achados apontaram prevalência de $24 \%$ para os comportamentos alimentares inadequados (EAT-26 $\geq 20$ ).

No que concerne as comparações do escore total e dos três fatores do EAT-26 de acordo com os grupos de estado nutricional, foram encontradas diferenças significativas nas subescalas dieta $(F(4,249)=28,55, p=0,001)$ e bulimia e preocupação com alimentos $(F(4,249)=19,34, p=$ 0,02 ; Tabela 1). Deste modo, os resultados evi- 
denciaram que as meninas com sobrepeso/obesidade demonstraram maior frequência de restrição alimentar e comportamentos purgativos quando comparadas as jovens classificadas com baixo peso e peso normal. Além disso, encontraram-se maiores pontuações do EAT-26 em adolescentes com sobrepeso e obesidade $(F(4,249)$ $=24,09, p=0,01$; Tabela 1).

\section{Tabela 1}

Comparação do Escore Total e dos Três Fatores do EAT-26 de Adolescentes do Sexo Feminino de Juiz de Fora segundo o Estado Nutricional

\begin{tabular}{lcccc}
\hline & \multicolumn{4}{c}{ Estado Nutricional } \\
\cline { 2 - 5 } Variável & Baixo Peso $(n=13)$ & Peso Normal $(n=196)$ & Sobrepeso $(n=26)$ & Obeso $(n=14)$ \\
& $M(D P)$ & $M(D P)$ & $M(D P)$ & $M(D P)$ \\
\hline Dieta & $2,87(1,93)^{* * *, * * * *}$ & $6,91(0,73)^{* * *, * * *}$ & $13,62(1,15)$ & $14,46(1,82)$ \\
Bulimia e PA & $1,71(0,53)$ & $1,77(0,22)$ & $3,49(0,72)$ & $4,14(0,59)^{*, * *}$ \\
Autocontrole O & $5,15(0,85)$ & $4,37(0,39)$ & $4,29(0,78)$ & $5,08(0,66)$ \\
EAT-26 (ET) & $10,18(2,40)^{* * *, * * *}$ & $12,61(0,69)^{* * *, * * * *}$ & $21,07(1,53)$ & $24,93(2,98)$ \\
\hline
\end{tabular}

Notas. $M=$ média; $D P=$ desvio padrão; $\mathrm{PA}=$ preocupação com alimentos; $\mathrm{O}=$ oral; $\mathrm{ET}=$ Escore total.

${ }^{*} p<0,05$ em relação a "Baixo Peso; ${ }^{* *} p<0,05$ em relação a "Peso Normal"; ${ }^{* * *} p<0,05$ em relação a "Sobrepeso"; ${ }^{* * * *} p$ $<0,05$ em relação a Obeso.

Em relação à razão de chances para o comportamento alimentar inadequado, o modelo de regressão logística demonstrou associação com o estado nutricional $\left(\right.$ Wald $=28,68, X^{2}(1,249)=$ $31,94, p=0,03)$. Logo, os resultados apontaram que as meninas com sobrepeso e obesidade demonstraram, respectivamente, 2,14 e 3,29 vezes mais chances para os comportamentos alimentares inadequados quando comparadas as meninas eutróficas, conforme ilustrado na Tabela 2.

Tabela 2

Razão de Chances para Comportamento Alimentar Inadequado (categoria de referência: EAT $<20)$ em adolescentes do sexo feminino de Juiz de Fora segundo o Estado Nutricional

\begin{tabular}{clcc}
\hline Variável & \multicolumn{1}{c}{ Classificação } & OR & IC (95\%) \\
\hline \multirow{2}{*}{ EN (IMC) } & Baixo peso & 0,87 & $0,55-4,81$ \\
& Peso Normal & 1,00 & - \\
& Sobrepeso & 2,14 & $1,56-6,13$ \\
& Obesidade & 3,29 & $1,97-9,20$ \\
\hline
\end{tabular}

Nota . EN = Estado Nutricional; IMC = Índice de massa corporal; OR* = Razão de chances (odds ratio); IC = Intervalo de Confiança.

\section{Discussão}

O estudo teve como premissa comparar os fatores do comportamento alimentar inadequado em razão do estado nutricional em adolescentes do sexo feminino de Juiz de Fora/MG. Os resultados evidenciaram que as adolescentes classificadas com sobrepeso/obesidade apresen- taram pontuações nas subescalas dieta, bulimia e preocupação com alimentos e no EAT-26 maiores comparadas as jovens com baixo peso/ peso normal.

Evidências indicam que as jovens do sexo feminino se restringem com frequência a alimentos com alto teor calórico (Gomes et al., 2010; Martins et al., 2010). Talvez, este comportamen- 
to esteja associado à maior depreciação com o peso e a aparência física, geralmente encontrada nesse sexo (Duca et al., 2010; Miranda et al., 2011). Em adição, as meninas adolescentes parecem estar mais susceptíveis para as influências advindas de pais e amigos do que os meninos (Alvarenga et al., 2011; Bernardi et al., 2009). Parece que as meninas adolescentes costumam apresentar mais adaptações psicológicas negativas relacionadas aos comentários depreciativos sobre o peso e a aparência corporal, normalmente advindos de familiares e grupos sociais, os quais estas jovens estão inseridas (Rossi et al., 2008). Talvez, por isso, meninas desenvolvam comportamentos alimentares deletérios a saúde com o intuito de remodelamento corporal, na tentativa de serem aceitas socialmente (Alvarenga et al., 2011; Martins et al., 2010; Scherer et al., 2010).

Remetendo-se à comparação das subescalas doEAT-26 segundo o estado nutricional, os resultados da presente investigação evidenciaram que as adolescentes com sobrepeso/obesidade apresentaram maior frequência de recusa alimentar e preocupação com a forma física quando comparadas a jovens com baixo peso/peso normal. Parece que a cultura do corpo magro, disseminada pela mídia e pelos meios de comunicação em massa, afeta mais as adolescentes que apresentam excesso de peso (Alvarenga et al., 2011; Gomes et al., 2010; Petroski, Pelegrini, \& Glaner, 2009; Scherer et al., 2010). Talvez, por isso, as jovens com sobrepeso/obesidade preocupem-se mais com o peso e a aparência física. No entanto, a recusa alimentar mais frequente em relação as adolescentes com baixo peso/peso normal não se justifica. Provavelmente por a subescala dieta do EAT-26 não separar a avaliação dos dois quesitos (recusa alimentar e preocupação com a forma física), torna-se inadequado falar que adolescentes com sobrepeso/obesidade apresentem recusa alimentar mais frequente quando comparadas com as jovens eutróficas. Contudo, as meninas classificadas com obesidade demonstraram maior frequência de comportamentos purgativos quando comparadas as meninas com baixo peso/ peso normal. Estes achados estão de acordo com o que a literatura preconiza, ou seja, as jovens com sintomas de bulimia costumam apresentar excesso de peso (Alvarenga et al., 2011; Duca et al., 2010; Martins et al., 2010; Rossi et al., 2008). Em contrapartida, não foi encontrada diferença na subescala autocontrole oral entre os grupos de estado nutricional, contrapondo as hipóteses apresentadas por outros autores de que as jovens com sobrepeso/obesidade costumam sofrer maiores pressões de familiares e amigos a respeito da redução do peso corporal (Bernardi et al., 2009; Gomes et al., 2010; Pelegrini \& Petroski, 2009). Talvez, as influências negativas parentais e de grupos sociais sejam semelhantes entre as adolescentes com diferentes estados nutricionais na cidade de Juiz de Fora/MG.

O modelo de regressão logística, por sua vez, demonstrou associação do comportamento alimentar inadequado com o estado nutricional. Neste sentido, os achados indicaram que as meninas com sobrepeso e obesidade apresentaram riscos aumentados para o desencadeamento dos comportamentos alimentares inadequados. Outras investigações científicas corroboram estes resultados (Fortes, Almeida, \& Ferreira, 2012a; Scherer et al., 2010). De fato, as jovens com excesso de peso estão mais vulneráveis para adotarem condutas alimentares deletérias a saúde com o propósito de emagrecimento. Alguns autores apontam a mídia como a principal responsável por esta situação (Amaral, Cordás, Conti, \& Ferreira, 2011; Fortes, Oliveira, \& Ferreira, 2012b). A transmissão de imagens de modelos e atrizes com o delineamento corporal magro faz com que as meninas mais gordinhas internalizem em suas mentes que a magreza é requisito essencial para a aceitação social. Deste modo, a gordura corporal é considerada aspecto morfológico depreciado na cultura ocidental (Fortes et al., 2012a). No entanto, de acordo com Scherer et al. (2010), a etapa da adolescência induz o aumento de gordura corporal no sexo feminino. Sendo assim, o quadro epidemiológico dos comportamentos alimentares inadequados no sexo feminino pode ser ainda mais alarmante.

O presente estudo demonstrou resultados inéditos e interessantes para o campo científico. No entanto, esta pesquisa apresentou limitações que merecem ser apontadas. Em primeiro lugar, a utilização de uma escala autoaplicável para avaliar comportamentos alimentares inadequados. Alguns autores ressaltam que as ferra- 
mentas auto reportadas podem mascarar a realidade do fenômeno no contexto avaliado, pois se tratam de respostas subjetivas. No entanto, Miranda et al. (2011) salientam que em estudos epidemiológicos ou de grandes amostras, os questionários são instrumentos que podem ser considerados "padrão ouro", pois não necessitam de mão de obra especializada para a sua aplicação, além de requisitar baixo custo operacional. A segunda limitação foi utilizar o IMC como ponto de corte para classificar o estado nutricional. Pesquisadores afirmam que o IMC pode classificar erroneamente sujeitos com massa muscular acentuada como obesos (Pelegrini $\&$ Petroski, 2009). Por outro lado, Castro et al. (2010) ressaltam que o IMC deve ser utilizado em pesquisas com tamanho amostral elevado, como é o caso da presente investigação. Por fim, o presente estudo evidenciou achados que merecem ser discutidos na literatura científica, pois se acredita que contemple análises que até então não haviam sido exploradas.

Os resultados da pesquisa permitem concluir que as jovens com sobrepeso/obesidade, residentes na cidade de Juiz de Fora/MG, apresentaram preocupação com a forma física e sintomas de bulimia mais frequentes quando comparadas ao grupo com baixo peso/peso normal.

Sugere-se que sejam realizados outros estudos com jovens brasileiros na tentativa de identificar possíveis fatores de risco para os comportamentos alimentares inadequados, investigando os demais fatores envolvidos na manifestação deste fenômeno.

\section{Referências}

Alvarenga, M. S., Scagliusi, F. B., \& Phillippi, S. T. (2011). Comportamento de risco para transtorno alimentar em universitárias brasileiras. Revista de Psiquiatria Clínica, 38(1), 3-7. doi: http:// dx.doi.org/10.1590/S0101-60832011000100002

Amaral, A. C. S., Cordás, T. A., Conti, M. A., \& Ferreira, M. E. C. (2011). Equivalência semântica e avaliação da consistência interna da versão em português do Sociocultural Attitudes Towards Appearance Questionnaire-3 (SATAQ-3). Cadernos de Saúde Pública, 27(8), 14871497. doi: http://dx.doi.org/10.1590/S0102$-311 \mathrm{X} 2011000800004$
Bernardi, F., Harb, A. N. C., Levandovski, R. M., \& Hidalgo, M. P. L. (2009). Transtornos alimentares e padrão circadiano alimentar - Uma revisão. Revista de Psiquiatria do Rio Grande do Sul, 31(3), 170-176. doi: http://dx.doi.org/10.1590/ S0101-81082009000300006

Bighetti, F., Santos, C. B., Santos, J. E., \& Ribeiro, R. P. P. (2004). Tradução e avaliação do Eating Attitudes Test em adolescentes do sexo feminino de Ribeirão Preto, São Paulo. Jornal Brasileiro de Psiquatria, 53(6), 339-346.

Castro, I. R. R., Levy, R. B., Cardoso, L. O., Passos, M. D., Sardinha, L. M. V., Tavares, L. F., ...Martins, A. (2010). Imagem corporal, estado nutricional e comportamento com relação ao peso entre adolescentes brasileiros. Ciência \& Saúde Coletiva, 15(Supl. 2), 3099-3108. doi: http:// dx.doi.org/10.1590/S1413-81232010000800014

Duca, G. F. D., Garcia, L. M. T., Sousa, T. F., Oliveira, E. S. A., \& Nahas, M. V. (2010). Insatisfação com o peso corporal e fatores associados em adolescentes. Revista Paulista de Pediatria, 28(4), 340-346. doi: http://dx.doi.org/10.1590/ S0103-05822010000400009

Dunker, K. L. L., Fernandes, C. P. B., \& Carreira, D., Filho. (2009). Influência do nível socioeconômico sobre comportamentos de risco para transtornos alimentares em adolescentes. Jornal Brasileiro de Psiquiatria, 58(3), 156-161. doi: http:// dx.doi.org/10.1590/S0047-20852009000300003

Fortes, L. S., Almeida, S. S., \& Ferreira, M. E. C. (2012a). Impacto de variáveis antropométricas sobre a insatisfação corporal e o comportamento alimentar em jovens atletas. Jornal Brasileiro de Psiquiatria, 61(4), 235-241. doi: http://dx.doi. org/10.1590/S0047-20852012000400007

Fortes, L. S., Oliveira, F. G., \& Ferreira, M. E. C. (2012b). Influência de fatores afetivos, antropométricos e sociodemográficos sobre o comportamento alimentar em jovens atletas. Jornal Brasileiro de Psiquiatria, 61(3), 148-153. doi: http:// dx.doi.org/10.1590/S0047-20852012000300005

Garner, D. M., Olmsted, M. P., Bohr, Y., \& Garfinkel, P. A. (1982). The eating attitudes test: Psychometric features and clinical correlations. Psychology Medicine, 12(1), 871-878.

Gomes, J. P., Legnani, E., Legnani, R. F. S., Gregorio, N. P., \& Souza, R. K. (2010). Associação entre comportamento alimentar, consumo de cigarro, drogas e episódios depressivos em adolescentes. Revista de Nutrição, 23(5), 755-762. doi: http:// dx.doi.org/10.1590/S1415-52732010000500006 
Johnson, C., Crosby, R., Engel, S., Mitchell, J., Powers, P., Wittrock, D., \& Wonderlich, S. (2004). Gender, ethnicity, self-esteem and disordered eating among college athletes. Eating Behaviors, 5(1), 147-156. doi: http://dx.doi.org/10.1016/j. eatbeh.2004.01.004

Martins, C. R., Pelegrini, A., Matheus, S. C., \& Petroski, E. L. (2010). Insatisfação com a imagem corporal e a relação com estado nutricional, adiposidade corporal e sintomas de anorexia e bulimia em adolescentes. Revista de Psiquiatria do Rio Grande do Sul, 32(1), 19-23. doi: http:// dx.doi.org/10.1590/S0101-81082010000100004

Miranda, V. P. N., Conti, M. A., Bastos, R., \& Ferreira, M. E. C. (2011). Insatisfação corporal em adolescentes brasileiros de municípios de pequeno porte de Minas Gerais. Jornal Brasileiro de Psiquiatria, 60(3), 190-197. doi: http://dx.doi. org/10.1590/S0047-20852011000300007

Oliveira, L. L., \& Hutz, C. S. (2010). Transtornos alimentares: O papel dos aspectos culturais no mundo contemporâneo. Psicologia em Estudo, 15(3), 575-582. doi: http://dx.doi.org/10.1590/ S1413-73722010000300015

Pelegrini, A., \& Petroski, E. L. (2009). Inatividade física e sua associação com estado nutricional, insatisfação com a imagem corporal e comportamentos sedentários em adolescentes de escolas públicas. Revista Paulista de Pediatria, 27(3), 366-373.

Petroski, E. L., Pelegrini, A., \& Glaner, M. F. (2009). Insatisfação corporal em adolescentes rurais e urbanos. Motricidade, 5(4), 13-25.

Rossi, A., Moreira, E. A. D., \& Rauen, M. S. (2008). Determinantes do comportamento alimentar Uma revisão com enfoque na família. Revista de Nutrição, 21(6), 739-748. doi: http://dx.doi. org/10.1590/S1415-52732008000600012
Santos, E. M. C., Tassitano, R. M., Nascimento, W. M. F., Petribu, M. M. V., \& Cabral, P. C. (2011). Satisfação com o peso corporal e fatores associados em adolescentes do ensino médio. Revista Paulista de Pediatria, 29(2), 214-223. doi: http://dx.doi.org/10.1590/S010305822011000200013

Scherer, F. C., Martins, C. R., Pelegrini, A., Matheus, S. C., \& Petroski, E. L. (2010). Imagem corporal em adolescentes: Associação com a maturação sexual e sintomas de transtornos alimentares. Jornal Brasileiro de Psiquiatria, 59(3), 198-202. doi: http://dx.doi.org/10.1590/S004720852010000300005

Siervogel, R. M., Dmerath, E. W., Sschubert, C., Remsberg, K. E., Chumlea, W. C., Sun, S., ... Towne, B. (2003). Puberty and body composition. Hormone Research, 60(1), 36-45.

World Health Organization. (2007). Development of a WHO growth reference for school-aged children and adolescents. Bulletin of the World Health Organization, 85(9), 660-667. doi: http:// dx.doi.org/10.2471/BLT.07.04349

Ximenez, R. C. C., Colares, V., Bertulino, T., Couto, G. B. L., \& Sougey, E. B. (2011). Versão brasileira do BITE para uso em adolescentes. Arquivos Brasileiros de Psicologia, 63(1), 52-63. 